PROCEEDINGS OF THE

AMERICAN MATHEMATICAL SOCIETY

Volume 140, Number 11, November 2012, Pages 4027-4034

S 0002-9939(2012)11224-9

Article electronically published on March 7, 2012

\title{
HOMOLOGY-GENERICITY, HORIZONTAL DEHN SURGERIES AND UBIQUITY OF RATIONAL HOMOLOGY 3-SPHERES
}

\author{
JIMING MA \\ (Communicated by Daniel Ruberman)
}

\begin{abstract}
In this paper, we show that rational homology 3-spheres are ubiquitous from the viewpoint of Heegaard splitting. Let $M=H_{+} \cup_{F} H_{-}$be a genus $g$ Heegaard splitting of a closed 3-manifold and $c$ be a simple closed curve in $F$. Then there is a 3-manifold $M_{c}$ which is obtained from $M$ by horizontal Dehn surgery along $c$. We show that for $c$ such that the homology class $[c]$ is generic in the set of curve-represented homology classes $\mathscr{H}_{s} \subset H_{1}(F)$, $\operatorname{rank}\left(H_{1}\left(M_{c}, \mathbb{Q}\right)\right)<\max \left\{1, \operatorname{rank}\left(H_{1}(M, \mathbb{Q})\right\}\right.$. As a corollary, for a set of curves $\left\{c_{1}, c_{2}, \ldots, c_{m}\right\}, m \geq g$, such that each $\left[c_{i}\right]$ is generic in $\mathscr{H}_{s} \subset H_{1}(F)$, $M_{\left(c_{1}, c_{2}, \ldots, c_{m}\right)}$ is a rational homology 3 -sphere.
\end{abstract}

\section{INTRODUCTION}

Thurston conjectured 13 that any closed hyperbolic 3-manifold $M$ admits a finite cover $\widetilde{M}$ which has positive first Betti number. There has been little progress on this conjecture for more than 20 years (see, for example, [1, 2] and [7: a small list from the huge literature on this topic).

Why is this conjecture so difficult to confirm? One of the reasons is that in some sense, closed 3-manifolds with positive first Betti numbers are rare in the set of all closed 3-manifolds. For example, any closed 3-manifold $M$ can be obtained from $S^{3}$ by a Dehn surgery along a link $K$, and the resulting manifold having positive first Betti number implies a strong restriction on the Dehn surgery coefficient; see [10]. Due to the lack of uniqueness of the surgery descriptions of 3-manifolds, the above argument just shows that "most" closed 3-manifolds have zero first Betti numbers in a philosophical sense, but not rigorously.

There are notions of random 3-manifolds using triangulations, Heegaard splittings and mapping class groups 2], and we can study the set of all 3-manifolds wholly from the probabilistic point of view. Since the set of all 3-manifolds is an infinite countable set, the notion of probability for some property of the set of 3manifolds must be defined artificially, but naturally, for the sake of mathematical interest.

In this paper, we show that most closed 3-manifolds have zero first Betti numbers from the viewpoint of Heegaard splitting:

Received by the editors January 31, 2010 and, in revised form, June 3, 2010; September 17, 2010; March 4, 2011; and April 26, 2011.

2010 Mathematics Subject Classification. Primary 57M27, 57M99.

Key words and phrases. Rational homology 3 -sphere, Heegaard splitting, homology-genericity. The author was supported in part by RFDP 200802461001 and NSFC 10901038. 
Theorem 1.1. Let $M=H_{+} \cup_{F} H_{-}$be a genus $g \geq 2$ Heegaard splitting of a closed 3-manifold, for a simple closed curve $c$ in $F$ such that the homology class $[c]$ is generic in the set of curve-represented homology classes $\mathscr{H}_{s} \subset H_{1}(F)$. Then $\operatorname{rank}\left(H_{1}\left(M_{c}, \mathbb{Q}\right)\right)<\max \left\{1, \operatorname{rank}\left(H_{1}(M, \mathbb{Q})\right\}\right.$, where $M_{c}$ is the manifold obtained from $M$ by horizontal Dehn surgery along $c$ in the sense of $Y$. Moriah and J. Schultens.

For precise definitions of the genericity, curve-represented homology classes and $\mathscr{H}_{s}$, see Definition 2.1.

Corollary 1.2. Let $M=H_{+} \cup_{F} H_{-}$be a genus $g \geq 2$ Heegaard splitting of a closed 3-manifold. Then for a set of curves $\left\{c_{1}, c_{2}, \ldots, c_{m}\right\}, m \geq g$, such that each $\left[c_{i}\right]$ is generic in $\mathscr{H}_{s} \subset H_{1}(F), M_{\left(c_{1}, c_{2}, \ldots, c_{m}\right)}$ is a rational homology 3-sphere.

J. Johnson and T. Patel [4 introduced a set $\mathscr{B}^{\infty}$, called the generalized handlebody set, in the curve complex $\mathscr{C}(\partial H)$ for a handlebody $H$, and they proved that $M=H_{+} \cup_{F} H_{-}$has zero first Betti number if and only if the generalized handlebody sets $\mathscr{B}_{+}^{\infty}$ and $\mathscr{B}_{-}^{\infty}$ associated to $H_{+}$and $H_{-}$have empty intersection. For any fixed copy of $\mathscr{B}^{\infty}$ in $\mathscr{C}(F)$ given by an identification $F=\partial H$ for some handlebody $H$, it seems difficult to detect whether or not $\mathscr{B}^{\infty}$ and $f\left(\mathscr{B}^{\infty}\right)$ intersect, for an automorphism $f$ of $F$. However, we have the following:

Theorem 1.3. Let $\mathscr{B}^{\infty} \subset \mathscr{C}(F)$ be given by an identification $F=\partial H$ for some handlebody $H$. For a set of curves $\left\{c_{1}, c_{2}, \ldots, c_{m}\right\}, m \geq g$, such that each $\left[c_{i}\right]$ is generic in $\mathscr{H}_{s} \subset H_{1}(F)$, the set $D_{c_{1} c_{2} \cdots c_{m}}\left(\mathscr{B}^{\infty}\right)$ is disjoint from $\mathscr{B}^{\infty}$, where $D_{c_{1} c_{2} \ldots c_{m}}$ is the composition of the Dehn twists along $c_{1}, c_{2}, \ldots, c_{m}$ iteratively.

Remark 1.4. Our notion of the genericity in $H_{1}(F)$ is given with respect to a basis of $H_{1}(F)$ related to the Heegaard splitting $M=H_{+} \cup_{F} H_{-}$, which has to satisfy some specific conditions (which are however natural in the given context). For an arbitrarily chosen basis of $H_{1}(F)$, whether or not results analogous to ours hold is unknown to the author.

Remark 1.5. Dunfield and Thurston proved a result similar to Corollary 1.2, in Corollary 8.5 of [2, using random walks in the mapping class group of a surface. Our results can be viewed as reconfirming the notion that rational homology 3spheres are more common than the 3-manifolds that are not rational homology 3 -spheres.

\section{Preliminaries}

For a surface $F$, M. Lustig and Y. Moriah introduced the notion of a generic curve in [8, which is based on the Lebesgue measure of $\mathscr{P} \mathscr{M} \mathscr{F}(F)$ - the natural measure class on the projective measured foliation space induced by local charts, i.e., the measured foliations carried by bi-recurrent maximal train tracks. This notion is more natural than others.

For $M=H_{+} \cup_{F} H_{-}$a genus $g \geq 2$ Heegaard splitting of a closed 3-manifold, we can look at $M$ as gluing $H_{+}$and $H_{-}$by an orientation-reversing homeomorphism $\tau: \partial H_{+} \longrightarrow \partial H_{-}$. Letting $c$ be a simple closed curve in $F=\partial H_{-}$, we reglue $H_{+}$ and $H_{-}$by $D_{c} \circ \tau$, where $D_{c}$ is the right Dehn twist along $c$, and we denote the resulting manifold by $M_{c}$. Note that $M_{c}$ can also be looked at as obtained from $M$ by $1 / 1$ surgery along $c$, where the meridian-longitude coordinate is given by the 
meridian on $\partial N(c)$ and the "horizontal" slope $F \cap \partial N(c)$, i.e., the horizontal Dehn surgery in the sense of Y. Moriah and J. Schultens; see [9].

We fix a canonical homology basis $\alpha=\left\{\left[a_{1}\right],\left[a_{2}\right], \ldots,\left[a_{2 g}\right]\right\}$ of $H_{1}(F)$; i.e., $a_{1}, a_{2}, \ldots, a_{2 g}$ are simple closed curves in $F$, and $F-\left\{a_{1}, a_{2}, \ldots, a_{g}\right\}$ is a $2 g$ holed sphere, such that the geometric intersection number of $a_{i}$ and $a_{g+j}$ is $\delta_{i j}$. Then an element of $H_{1}(F)$ can be written as $\sum_{i=1}^{2 g} x_{i}\left[a_{i}\right], x_{i} \in \mathbb{Z}$. We denote by $|x|_{\alpha}=\max \left\{\left|x_{i}\right|, 1 \leq i \leq 2 g\right\}$, the $\alpha$-norm of the homology class $x$. This homology class can be represented by a simple closed curve in $F$ if and only if the greatest common denominator of $\left\{x_{1}, x_{2}, \ldots, x_{2 g}\right\}$ is 1 (see [11]), and if this is the case, we say that the homology class is curve-represented. Note that whether or not a homology class is curve-represented is independent of the choice of the homology basis of $H_{1}(F)$. Let $\mathscr{H}_{s}$ be the collection of all homology classes in $H_{1}(F)$ which are curve-represented.

Now let $c$ and $d$ be two simple closed curves in $F$ which represent the same homology class in $H_{1}(F)$. It is easy to see that $H_{1}\left(M_{c}\right)=H_{1}\left(M_{d}\right)$, for example, from the proof of Theorem 1.1. So the homology of the surgered manifold is a property preserved in the homology class. Thus, we introduce the notion of generic curve-represented homology classes:

Definition 2.1. For a property of points in $\mathscr{H}_{s}$, if the following holds:

$$
\lim _{N \rightarrow \infty} \frac{\sharp\left\{x \in \mathscr{H}_{s} \mid x \text { satisfies the property }|x|_{\alpha} \leq N\right\}}{\sharp\left\{\left.x \in \mathscr{H}_{s}|| x\right|_{\alpha} \leq N\right\}}=1,
$$

then we say that the property is satisfied for generic curve-represented homology classes with respect to the basis $\alpha=\left\{\left[a_{1}\right],\left[a_{2}\right], \ldots,\left[a_{2 g}\right]\right\}$ of $H_{1}(F)$.

A family of homology classes $\left\{\left[c_{i}\right]\right\}_{i=1}^{m}$ is called generic (in $\mathscr{H}_{s}$ ) if each class $\left[c_{i}\right]$ in the family is generic (in $\mathscr{H}_{s}$ ), by which we mean precisely: $\left[c_{i}\right]$ belongs to a subset of $\mathscr{H}_{s}$ such that the property "belonging to this subset" is generic in the above-defined sense.

Definition 2.2 (see [4). For a handlebody $H$ of genus $g$, let $\mathcal{B}_{i}$ be the set of non-separating simple closed curves in $\partial H$ that bound a genus $i$ incompressible surface with one boundary in $H$. Let $\mathscr{B}^{\infty}=\bigcup_{i=0}^{\infty} \mathcal{B}_{i}$. Note that $\mathcal{B}_{0}$ is just the set of non-separating meridians in $\partial H$. The union $\mathscr{B}^{\infty}$ is called the generalized handlebody set or the boundary set.

Recall that the curve complex $\mathscr{C}(F)$ for a closed surface $F$ of genus at least two is the graph whose vertices correspond to the isotopy classes of essential curves in $F$, and there is a length 1 edge between two vertices $a, b$ whenever the isotopy classes $a, b$ can be realized by disjoint curves in $F$.

J. Johnson and T. Patel 4 proved that $\mathscr{B}^{\infty}$ is 2-dense in $\mathscr{C}(\partial H)$ if $H$ has genus at least three. By this we mean that for any essential simple closed curve $c$ in $\partial H$, there is an essential simple closed curve $b$ in $\mathscr{B}^{\infty}$ such that the distance between $c$ and $b$ in $\mathscr{C}(\partial H)$ is at most two. Lemma 5.10 of S. Schleimer 12 implies that $\mathscr{B}^{\infty}$ is 5-dense in $\mathscr{C}(\partial H)$ if $H$ has genus two. Thus it is difficult to separate the boundary set from its $f$-image, for an automorphism $f$ of $F$.

One of the potential approaches to show the ubiquity of rational homology 3spheres is to show that the closure of $\mathscr{B}^{\infty}$ is small in $\mathscr{P} \mathscr{M} \mathscr{F}(F)$, where $F$ is the boundary of a handlebody $H$. Recall that S. Kerckhoff [6] proved that the closure of $\mathcal{B}_{0}$ in $\mathscr{P} \mathscr{M} \mathscr{F}(F)$ has zero measure. But the following proposition blocks this approach: 
Proposition 2.3. For a handlebody $H$ of genus $g \geq 2$, the closure of $\mathscr{B}^{\infty}$ in $\mathscr{P} \mathscr{M} \mathscr{F}(\partial H)$ is $\mathscr{P} \mathscr{M} \mathscr{F}(\partial H)$.

Proof. We first assume that the genus of $H$ is at least three. Let $\lambda$ be a minimal uniquely ergodic foliation in $\partial H$. Then there is a sequence of simple closed curves $\left\{a_{i}\right\}_{i=1}^{\infty}$ in $\partial H$ which converges to $\lambda$ in $\mathscr{P} \mathscr{M} \mathscr{F}(\partial H)$. But by Klarreich's result on the boundary of the curve complex [5], we have that $\left\{a_{i}\right\}$ converges to $\lambda$ in $\mathscr{C}(\partial H)$, where $\lambda$ is understood as a point of the Gromov boundary of the curve complex. Since $\mathscr{B}^{\infty}$ is 2 -dense in the curve complex, there is $b_{i} \in \mathscr{B}^{\infty}$ with $d_{\mathscr{C}(\partial H)}\left(a_{i}, b_{i}\right) \leq 2$. So $\left\{b_{i}\right\}$ converges to $\lambda$ also; see Lemma 3.1 of [3]. Then, by Masur's result that almost every foliation is uniquely ergodic, the closure of $\mathscr{B}^{\infty}$ is $\mathscr{P} \mathscr{M} \mathscr{F}(\partial H)$.

The proof of the genus two case is similar, just replacing the 2-density of $\mathscr{B}^{\infty}$ in the curve complex by the 5 -density.

The other potential approach is using the structure of the mapping class group $\mathcal{M C G}(F)$ and its dynamic properties. But note that the Torelli group $\mathscr{T}_{g}$, the subgroup of $\mathcal{M C G}(F)$ consisting of elements that act trivially on $H_{1}(F)$, has infinite index in $\mathcal{M C G}(F)$, and it is well known that the limit set of $\mathscr{T}_{g}$, say $\Lambda\left(\mathscr{T}_{g}\right)$, is the full space $\mathscr{P} \mathscr{M} \mathscr{F}(F)$ (for example, from Fact 3.2 ), so this approach is difficult, and it seems this approach always fails.

\section{Proof of the theorems}

In this section we prove our theorems.

Proof of Theorem 1.1. Let $M=H_{+} \cup_{F} H_{-}$be a genus $g$ Heegaard splitting of a closed 3-manifold. Then $H_{1}(M, \mathbb{Z})$ has a presentation matrix $X$ of size $g \times g$, and the first Betti number is the rank of $H_{1}(M, \mathbb{Q})$ : take $a_{1}, a_{2}, \ldots, a_{g}$ to be a set of oriented simple closed curves in $\partial H_{+}$, each of which bounds a disk in $H_{+}$, such that these disks decompose $H_{+}$into a 3 -ball; we say that $\left\{a_{1}, a_{2}, \ldots, a_{g}\right\}$ is a basis of $H_{+}$. Similarly we define $b_{1}, b_{2}, \ldots, b_{g}$ in $\partial H_{-}$. Let $a_{1}^{*}, a_{2}^{*}, \ldots, a_{g}^{*}$ be a set of curves dual to $a_{1}, a_{2}, \ldots, a_{g}$ in $\partial H_{+}: a_{i}^{*}$ is a simple closed curve such that the geometric intersection number of $a_{i}^{*}$ and $a_{j}$ is $\delta_{i j}$. So $\left\{a_{1}^{*}, a_{2}^{*}, \ldots, a_{g}^{*}\right\}$ is a basis for $H_{1}\left(H_{+}\right)$, and $H_{1}(M, \mathbb{Z})$ is obtained from $H_{1}\left(H_{+}\right)$by adding the relations $b_{1}, b_{2}, \ldots, b_{g}$. Then $H_{1}(M, \mathbb{Z})$ has the presentation matrix $X=\left(\left(a_{i}, b_{j}\right)\right)_{g \times g}$, where $(.,$.$) is the algebraic intersection number of two curves. Note that the rank of X$ is just $g-\operatorname{rank}\left(H_{1}(M, \mathbb{Q})\right)$. We denote by $a_{i}$ also the curve $\tau\left(a_{i}\right)$ in $F=\partial H_{-}$.

Let $c$ be a simple closed curve in $F$. Then in $M_{c}$ the curve $\widetilde{a_{i}}$ bounds a disk in $H_{+}$, where $\widetilde{a_{i}}$ is the curve obtained from $a_{i}$ by a right Dehn twist along $c$. Thus one has $\left(\widetilde{a_{i}}, b_{j}\right)=\left(a_{i}, b_{j}\right)+\left(a_{i}, c\right)\left(c, b_{j}\right)$. If $d$ is another simple closed curve in $F$ such that $[c]=[d]$ in $H_{1}(F)$, then $\left(a_{i}, c\right)\left(c, b_{j}\right)=\left(a_{i}, d\right)\left(d, b_{j}\right)$, so $H_{1}\left(M_{c}\right)$ and $H_{1}\left(M_{d}\right)$ have the same presentation matrix. Thus $M_{c}$ and $M_{d}$ have the same homology.

Starting from $X$, we can transform it iteratively into $Y=\operatorname{diag}\left\{\lambda_{1}, \lambda_{2}, \ldots \lambda_{r}, 0\right.$, $\ldots, 0\}$, where $\lambda_{i} \mid \lambda_{i+1}$ are non-zero integers, by the following operations:

(1) interchange two rows or two columns,

(2) multiply a column (row) by -1 ,

(3) add to any column (row) an integer linear combination of other columns (rows).

Notice that the transformation from $X$ to $Y$ can be induced by the changes of the basis of $H_{+}$and the basis of $H_{-}$: transformation (1) is induced by a relabeling of the 


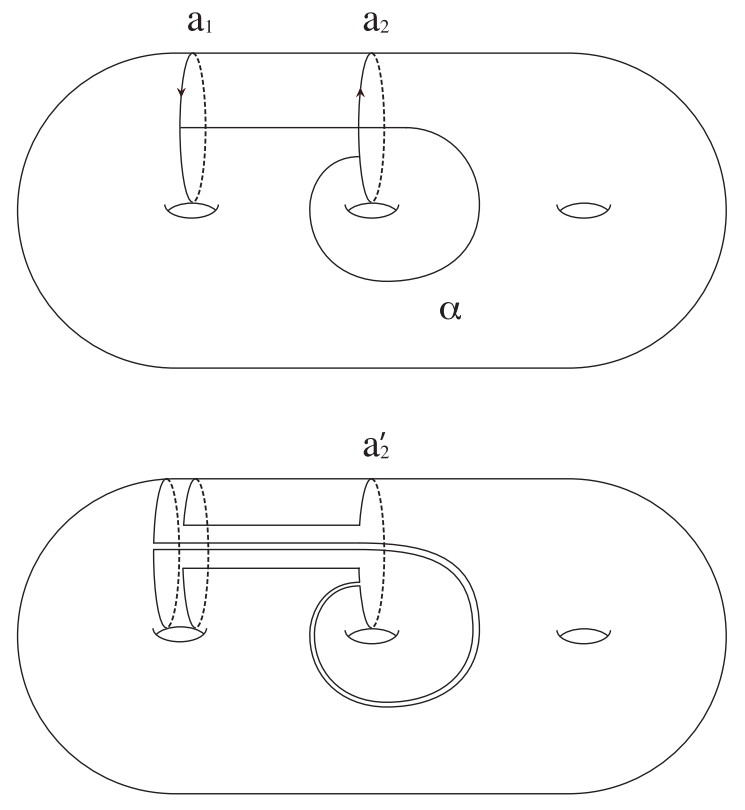

FiguRE 1

basis $\left\{a_{i}\right\}_{i=1}^{g}$ or $\left\{b_{i}\right\}_{i=1}^{g}$; transformation (2) is induced by reversing the orientation of one of $a_{i}$ or $b_{i}$; transformation (3) is induced by band-sums of disks: for example, corresponding to adding $k$ times the first column to the second column, we can take an oriented arc $\alpha$ from $\partial_{1} \alpha=\alpha \cap a_{1}$ to $\partial_{2} \alpha \in \alpha \cap a_{2}$, and $\left(a_{1}, \alpha\right)=1,\left(\alpha, a_{2}\right)=k$ (note that $k$ may be negative). We assume that $\alpha \cap a_{2}$ are $|k|$ points and we denote them by $p_{1}, p_{2}, \ldots, p_{|k|}$, where $p_{|k|}=\partial_{2} \alpha$ and we also denote $\partial_{1} \alpha$ by $p_{0}$. We can also assume $\alpha \cap a_{i}=\emptyset$ for $i \neq 1,2$. Then performing the disk-sum of $a_{1}$ and $a_{2}$ along the sub-arc $p_{0} p_{1}$, we get a disk $a_{1} \sharp_{p_{0}} p_{1} a_{2}$, which is disjoint from $a_{i}$ for $i \neq 2$, and $\alpha$ intersects $a_{1} \sharp_{p_{0} p_{1}} a_{2}$ in $p_{2}, p_{3}, \ldots, p_{|k|}$. Then we perform a disk-sum of $a_{1}$ and $a_{1} \sharp_{p_{0} p_{1}} a_{2}$ along $p_{0} p_{2}$. We also obtain a disk which is disjoint from $a_{i}$ for $i \neq 2$. So performing disk-sums $k$ times we get a disk $a_{2}^{\prime}$ such that $a_{1}, a_{2}^{\prime}, \ldots, a_{g}$ is a new basis of $H_{+}$. The intersection matrix of $a_{1}, a_{2}^{\prime}, \ldots, a_{g}$ and $b_{1}, b_{2}, \ldots, b_{g}$ corresponds to adding $k$ times the first column to the second column. See Figure 1 for the case of adding twice the first column to the second column.

In other words, we can rechoose the basis $\left\{a_{1}, a_{2}, \ldots, a_{g}\right\}$ of $H_{+}$and the basis $\left\{b_{1}, b_{2}, \ldots, b_{g}\right\}$ of $H_{-}$, such that $\left(\left(a_{i}, b_{j}\right)\right)_{g \times g}=\operatorname{diag}\left\{\lambda_{1}, \lambda_{2}, \ldots \lambda_{r}, 0, \ldots, 0\right\}$ and $H_{1}(M)=\mathbb{Z}^{g-r} \oplus \mathbb{Z}_{\lambda_{1}} \oplus \mathbb{Z}_{\lambda_{2}} \oplus \cdots \oplus \mathbb{Z}_{\lambda_{r}}$.

We consider

$$
\mathbf{S}=\left(\begin{array}{cc}
0 & I_{g} \\
-I_{g} & 0
\end{array}\right)
$$

Now $\left\{\left[a_{1}\right],\left[a_{2}\right], \ldots,\left[a_{g}\right],\left[a_{1}^{*}\right],\left[a_{2}^{*}\right], \ldots,\left[a_{g}^{*}\right]\right\}$ is a standard homology basis of $H_{1}(F)$, and we assume $a_{1}=(1,0,0 \ldots, 0), a_{2}=(0,1,0 \ldots, 0), \ldots, a_{g}=(0, \ldots$, $0,1 \mid 0,0 \ldots, 0)$ are $2 g$-dimensional row vectors, and $a_{1}^{*}=(0,0,0 \ldots, 0 \mid 1,0,0 \ldots, 0)$, $a_{2}^{*}=(0,0,0 \ldots, 0 \mid 0,1, \ldots, 0), \ldots, a_{g}^{*}=(0,0,0 \ldots, 0 \mid 0,0, \ldots, 1)$ are $2 g$-dimensional row vectors. 
For

$$
\mathbf{A}=\left(\begin{array}{ll}
I & 0
\end{array}\right)_{g \times 2 g},
$$

the vector $a_{i}$ is the $i$-th row vector of $A$. Let $B$ be a $g \times 2 g$ matrix such that the $i$-th row vector of $B$ is $b_{i}$ in the coordinate given by $\left\{\left[a_{1}\right],\left[a_{2}\right], \ldots,\left[a_{g}\right],\left[a_{1}^{*}\right],\left[a_{2}^{*}\right], \ldots,\left[a_{g}^{*}\right]\right\}$. Then the intersection matrix $\left(\left(a_{i}, b_{j}\right)\right)_{g \times g}$ is just $A S B^{T}$, where $B^{T}$ denotes the transposed matrix of $B$.

Now assume that $B=\left(B_{1}, B_{2}\right)$, where $B_{1}$ and $B_{2}$ are two $g \times g$ matrixes. Then we have $A S B^{T}=\operatorname{diag}\left\{\lambda_{1}, \lambda_{2}, \ldots, \lambda_{r}, 0, \ldots, 0\right\}$. So from the above claim, we have $\left(B_{2}^{T}\right)=\operatorname{diag}\left\{\lambda_{1}, \lambda_{2}, \ldots, \lambda_{r}, 0, \ldots, 0\right\}=B_{2}$. Denote by $\beta_{1}, \beta_{2}, \ldots, \beta_{g}$ the row vectors of $B_{1}$. Note that since $B=\left(B_{1}, B_{2}\right)$ is represented by $g$ homology independent curves in $F$, the rank of $B=\left(B_{1}, B_{2}\right)$ is $g$. Hence the bottom submatrix of $B_{1}$ which consists of $\beta_{r+1}, \beta_{r+2}, \ldots, \beta_{g}$ has rank $g-r$. In particular, each $\beta_{j}$ is non-zero, for $r+1 \leq j \leq g$ if $r<g$.

Let $[c]=\left(x_{1}, x_{2}, \ldots, x_{g}, y_{1}, \ldots, y_{g}\right)=(x, y)$ be a homology class with respect to the coordinate $\left\{\left[a_{1}\right],\left[a_{2}\right], \ldots,\left[a_{g}\right],\left[a_{1}^{*}\right],\left[a_{2}^{*}\right], \ldots,\left[a_{g}^{*}\right]\right\}$ that can be represented by a simple closed curve $c$ in $F$, where $x$ and $y$ are two $g$-dimensional row vectors.

Consider the presentation matrix $\widetilde{X}$ for $H_{1}\left(M_{c}, \mathbb{Z}\right)$, which is $\widetilde{X}=\left(\left(\widetilde{a_{i}}, b_{j}\right)\right)_{g \times g}=$ $\left(\left(a_{i}, b_{j}\right)+\left(a_{i}, c\right)\left(c, b_{j}\right)\right)_{g \times g}$. Note that $\left(a_{i}, c\right)=y_{i}$, and $\left(c, b_{j}\right)=x_{j} \lambda_{j}-\beta_{j} \cdot y$ for $1 \leq j \leq r$ and $\left(c, b_{j}\right)=-\beta_{j} \cdot y$ for $r+1 \leq j \leq g$, where $\beta_{j} \cdot y$ is the inner product of two vectors $\beta_{j}$ and $y$.

Now consider the left-top $(r+1) \times(r+1)$ submatrix of $\tilde{X}$, which is:

$$
\left(\begin{array}{cccc}
\lambda_{1}+y_{1}\left(x_{1} \lambda_{1}-\beta_{1} \cdot y\right) & \cdots & 0+y_{1}\left(x_{r} \lambda_{1}-\beta_{r} \cdot y\right) & 0+y_{1}\left(-\beta_{r+1} \cdot y\right) \\
\vdots & \vdots & \vdots & \vdots \\
0+y_{r}\left(x_{1} \lambda_{1}-\beta_{1} \cdot y\right) & \cdots & \lambda_{r}+y_{r}\left(x_{r} \lambda_{1}-\beta_{r} \cdot y\right) & 0+y_{r}\left(-\beta_{r+1} \cdot y\right) \\
0+y_{r+1}\left(x_{1} \lambda_{1}-\beta_{1} \cdot y\right) & \cdots & 0+y_{r+1}\left(x_{r} \lambda_{1}-\beta_{r} \cdot y\right) & 0+y_{r+1}\left(-\beta_{r+1} \cdot y\right)
\end{array}\right) \text {. }
$$

Thus, if $-\beta_{r+1} \cdot y \neq 0$, using the last column, we can simplify the first $r$ columns such that the determinant of it is $\lambda_{1} \cdots \lambda_{r} y_{r+1}\left(-\beta_{r+1} \cdot y\right)$, which is non-zero if $y_{r+1}$ is non-zero.

Note that the solution space of

$$
y_{r+1}\left(-\beta_{r+1} \cdot y\right)=0
$$

is just the union of two hyperplanes in $\mathbb{R}^{g}$, since $\beta_{r+1}$ is a non-zero vector.

Thus, if one of $y_{r+1}\left(-\beta_{r+1} \cdot y\right), \ldots, y_{g}\left(-\beta_{g} \cdot y\right)$ is non-zero, then $\widetilde{X}$ has an $(r+1) \times(r+1)$ submatrix which has non-zero determinant, which implies $\operatorname{rank}(\tilde{X})>$ $\operatorname{rank}(X)$. But note that $\beta_{r+1}, \beta_{r+2}, \ldots, \beta_{g}$ are linearly independent. The set of $\left(x_{1}, x_{2}, \ldots, x_{g}, y_{1}, \ldots, y_{g}\right)=(x, y) \in \mathbb{Z}^{2 g}$ such that all $y_{r+1}\left(-\beta_{r+1} \cdot y\right), \ldots, y_{g}\left(-\beta_{g}\right.$. $y)$ are zeros has measure zero in $\mathbb{Z}^{2 g}$. We are done.

If $r=g$, then $\widetilde{X}$ is given by

$$
\left(\begin{array}{cccc}
\lambda_{1}+y_{1}\left(x_{1} \lambda_{1}-\beta_{1} \cdot y\right) & \cdots & y_{1}\left(x_{g-1} \lambda_{1}-\beta_{g-1} \cdot y\right) & y_{1}\left(-\beta_{g} \cdot y\right) \\
\vdots & \vdots & \vdots & \vdots \\
y_{g-1}\left(x_{1} \lambda_{1}-\beta_{1} \cdot y\right) & \cdots & \lambda_{g-1}+y_{g-1}\left(x_{g-1} \lambda_{1}-\beta_{g-1} \cdot y\right) & y_{g-1}\left(-\beta_{g} \cdot y\right) \\
y_{g}\left(x_{1} \lambda_{1}-\beta_{1} \cdot y\right) & \cdots & y_{g}\left(x_{g-1} \lambda_{1}-\beta_{g-1} \cdot y\right) & \lambda_{g}+y_{g}\left(-\beta_{g} \cdot y\right)
\end{array}\right),
$$

so that the determinant of it is a polynomial in $x_{1}, x_{2}, \ldots, x_{g}, y_{1}, \ldots, y_{g}$, with zero degree coefficient $\lambda_{1} \lambda_{2} \cdots \lambda_{g}$. Hence the polynomial is non-zero and the set 
of $\left\{x_{1}, x_{2}, \ldots, x_{g}, y_{1}, \ldots, y_{g}\right\} \in \mathbb{Z}^{2 g}$ such that the determinant of $\tilde{X}$ is zero has measure zero in $\mathbb{Z}^{2 g}$.

Proof of Corollary 1.2. Now from Theorem 1.1, if $\operatorname{rank}\left(H_{1}(M, \mathbb{Q})\right)=r \neq 0$, then for a homology-generic curve $c, \operatorname{rank}\left(H_{1}\left(M_{c}, \mathbb{Q}\right)\right)=\operatorname{rank}\left(H_{1}(M, \mathbb{Q})\right)-1$. Thus by performing iteratively $r$ times horizontal Dehn surgeries along $c_{1}, c_{2}, \ldots, c_{r}$, we obtain that $M_{\left(c_{1}, c_{2}, \cdots c_{r}\right)}$ is a rational homology sphere, and for any homology-generic curve $c_{r+1}$, the manifold $M_{\left(c_{1}, c_{2}, \cdots c_{r+1}\right)}$ is also a rational homology sphere by Theorem 1.1 .

Remark 3.1. For two links $K_{1}$ and $K_{2}$ in $S^{3}$, it is difficult to show the relationship between the surgered manifolds along $K_{1}$ and $K_{2}$. For Heegaard splittings of manifolds as in Theorem 1.1, it can be shown that most different horizontal Dehn surgeries result in different manifolds: the main result of [8] by M. Lustig and Y. Moriah is that for any fixed number $n$, then for a generic curve $c$ (this notion is defined in terms of the Lebesgue measure on $\mathscr{P} \mathscr{M} \mathscr{F}(F)$ ), the manifold $M_{c}$ has Heegaard distance at least $n$. Hence for generic and homology-generic curves $\left\{c_{m+1}, \ldots, c_{k}\right\}$, the Heegaard distance of $M_{\left(c_{1}, c_{2}, \ldots, c_{k}\right)}$ is greater than the Heegaard distance of $M_{\left(c_{1}, c_{2}, \ldots c_{m}\right)}$ by Fact 3.2 below. Thus $M_{\left(c_{1}, c_{2}, \ldots c_{k}\right)}$ is not homeomorphic to $M_{\left(c_{1}, c_{2}, \ldots c_{m}\right)}$, for generic and homology-generic curves $c_{m+1}, \ldots, c_{k}$, if $k>m$.

Fact 3.2. For any $x$ in $\mathscr{H}_{s}$, i.e., a homology class in $H_{1}(F)$ which can be realized by a simple closed curve in $F$, the closure of the set $S_{x}$ of simple closed curves in $F$ that represent $x$ is the full space $\mathscr{P} \mathscr{M} \mathscr{F}(F)$.

Proof. This fact follows from the fact that the Torelli group $\mathscr{T}_{g}$ acts minimally on $\mathscr{P} \mathscr{M} \mathscr{F}(F)$. We include here a simple argument for completeness: take any $c$ in $S_{x}$, and take a separating curve $d$ which intersects $c$ essentially. Then $D_{d}^{n}(c)$ is homologous to $c$ in $H_{1}(F)$, and the limit of $D_{d}^{n}(c)$ in $\mathscr{P} \mathscr{M} \mathscr{F}(F)$ for $n \rightarrow \infty$ is $d$. Since separating curves are 1-dense in $\mathscr{C}(F)$, the claim follows from the arguments in Proposition 2.2.

Proof of Theorem 1.3. Fix a genus $g$ handlebody $H$. The double of $H$ is $N=$ $\sharp^{g} S^{2} \times S^{1}$, which has first Betti number $g$. For the genus $g$ Heegaard splitting $N=H_{+} \cup_{F} H_{-}$, we have $\mathscr{B}_{+}^{\infty}=\mathscr{B}_{-}^{\infty}$. We use Corollary 1.2 to obtain that $N_{\left(c_{1}, c_{2}, \ldots c_{m}\right)}$ is a rational homology sphere. Note that $N_{\left(c_{1}, c_{2}, \ldots c_{m}\right)}$ is obtained from $H_{+}$by gluing the mirror copy $H_{-}$of it with the gluing map $D_{c_{1} c_{2} \ldots c_{m}}$. By [4, we have that $D_{c_{1} c_{2} \ldots c_{m}}\left(\mathscr{B}_{+}^{\infty}\right)$ is disjoint from $\mathscr{B}_{-}^{\infty}=\mathscr{B}_{+}^{\infty}$.

\section{ACKNOWLEDGEMENTS}

The anonymous referee has generously done a lot of work on the text, both on mathematical content and on the English writing. The author appreciates the referee's valuable comments, corrections and suggestions.

\section{REFERENCES}

1. N. Dunfield and W. Thurston, The virtual Haken conjecture: experiments and examples, Geom. \& Topol. 7 (2003), 399-441. MR 1988291 (2004i:57024)

2. N. Dunfield and W. Thurston, Finite covers of random 3-manifolds, Invent. Math. 166(2006), 457-521. MR2257389 (2007f:57039)

3. U. Hamenstädt, Train tracks and the Gromov boundary of the complex of curves, Spaces of Kleinian groups (Y. Minsky, M. Sakuma and C. Series, eds.), London Math. Soc. Lec. Notes 329(2005), 187-207. MR2258749 (2009a:30092) 
4. J. Johnson and T. Patel, Generalized handlebody sets and non-Haken 3-manifolds, Pacific Journal of Math. 235(2008), 35-41. MR.2379768 (2008m:57043)

5. E. Klarreich, The boundary at infinity of the curve complex and the relative Teichmüller space, unpublished manuscript, Ann Arbor, 1999.

6. S. Kerckhoff, The measure of the limit set of the handlebody group, Topology 29(1990), 27-40. MR.1046623 (91b:57011)

7. M. Lackenby, Heegaard splittings, the virtually Haken conjecture and property $(\tau)$, Invent. Math. 164(2006), 317-359. MR2218779 (2007c:57030)

8. M. Lustig and Y. Moriah, Horizontal Dehn surgery and genericity in the curve complex, Journal of Topology 3(2010), 691-712. MR2684517

9. Y. Moriah and J. Schultens, Heegaard splittings of Seifert fibred spaces are either vertical or horizontal, Topology 37(1998), 1089-1112. MR1650355 (99g:57021)

10. D. Rolfsen, Knots and links, Berkeley: Publish or Perish Inc., 1976. MR0515288 (58:24236)

11. J. Schafer, Representing homology classes on surfaces, Canad. Math. Bull. 19(1976), 373-374. MR0454995 (56:13236)

12. S. Schleimer, Notes on the complex of curves, unpublished, 2006.

13. W. Thurston, On the geometry and dynamics of diffeomorphisms of surfaces, Bull. Amer. Math. Soc. (N.S.) 19(1988), 417-431. MR.956596 (89k:57023)

School of Mathematical Sciences, Fudan University, Shanghai, People's Republic of CHina 200433

E-mail address: majiming@fudan.edu.cn 